\title{
Postexperience Advertising Effects on Consumer Memory
}

\author{
KATHRYN A. BRAUN *
}

\begin{abstract}
Past research suggests that marketing communications create expectations that influence the way consumers subsequently learn from their product experiences. Since postexperience information can also be important and is widespread for established goods and services, it is appropriate to ask about the cognitive effects of these efforts. The postexperience advertising situation is conceptualized here as an instant source-forgetting problem where the language and imagery from the recently presented advertising become confused with consumers' own experiential memories. It is suggested that, through a reconstructive memory process, this advertising information affects how and what consumers remember. Consumers may come to believe that their past product experience had been as suggested by the advertising. Over time this postexperience advertising information can become incorporated into the brand schema and influence future product decisions.
\end{abstract}

$\mathrm{C}$ onsumers perceive their own experiences as special, and direct experiences are generally thought to be an integral part of how consumers learn objective and affective responses to products (Fazio, Zanna, and Cooper 1978; Hoch and Deighton 1989; Holbrook and Hirschman 1982; Smith 1993; Smith and Swinyard 1983). However, the objectivity of these learning experiences has been questioned. It has been suggested that marketing communications can create expectations that influence the way consumers subsequently learn from their experience (Hoch and Deighton 1989, p. 1): "Learning from selfgenerated experience with a product or service is not a simple process of discovering objective truth. It is, to a greater extent, open to influence, and the consumer's confidence in the objectivity of such learning can be illusory."

The effects of experiential learning on consumer decision making may not occur until some time after the

*This research is based on the author's dissertation, "Memory Reconstruction in Consumer Decision Making," conducted at the University of Iowa. She would like to thank committee members for their help in developing this idea, especially Gary Gaeth who served as chair, Lola Lopes for her question that led to experiment 1, Baba Shiv for his comments on a previous draft, and Gerald Zaltman for his support and feedback throughout this process. The author thanks several others who also had a great impact on this article: Dan Schacter, for suggesting how to approach alternative explanations; Elizabeth Loftus, for design ideas and feedback on this manuscript; Dan Wright, for comments on retrieval processes; the reviewers; and especially the associate editor who provided guidance on developing this manuscript. Kathryn A. Braun is serving as a visiting scholar in the Mind of the Market Lab at the Harvard Business School. She can be reached via e-mail at kbraun@ hbs.edu. experience. It is therefore appropriate to inquire about the consequences of such learning. The paradox is that while experiential information is learned fast (Wright and Lynch 1995), this type of information is also the most fragile, context-dependent, and subject to distortion (Snodgrass 1997). The issue addressed here is whether advertising received after a direct product experience can alter how consumers come to remember their sensory and affective responses to that product. In essence, is it possible for marketers to reshape consumers' experiential memories (even bad ones) to become more favorable?

\section{CONCEPTUAL BACKGROUND}

Memory is an active constructive process where information is acquired, stored, and then retrieved for use in decision making (see Fig. 1). The majority of marketers' attention has been given to the left side of this diagram. A forward-framing theory has been advanced where prior information acts as a memory schema and influences the perception of a current experience (Alba and Hasher 1983; Bower, Black, and Turner 1979). Memory schemas can implicitly color the present learning environment by affecting how individual neurons code information (Merzenich and deCharms 1996). In marketing, these schemas have been found to affect how consumers interpret sensory product experiences. Examples of such schema effects can be found in the work of Allison and Uhl (1964), who concluded that a positive brand schema made beer taste better, and Olson and Dover (1979), who discovered suggestive advertising inoculated consumers so they enjoyed bitter coffee that they probably would not have tasted had it not been for the successful ad campaign. 
FIGURE 1

DYNAMIC MEMORY PROCESS

\begin{tabular}{|c|c|c|c|c|}
\hline \multicolumn{3}{|c|}{ Constructive Acquisition Processes } & \multirow{2}{*}{\multicolumn{2}{|c|}{$\begin{array}{l}\text { Reconstructive Retrieval Processes } \\
\text { Environmental cues }\end{array}$}} \\
\hline $\begin{array}{l}\text { Acquisition of } \\
\text { original exper } \\
\Downarrow\end{array}$ & $\begin{array}{l}\text { Acquisitic } \\
\text { nce } \\
\downarrow\end{array}$ & information & & \\
\hline $\begin{array}{l}\text { ADecision } \Rightarrow \\
\text { about what to } \\
\text { look at } \\
\text { influenced by } \\
\text { schema or } \\
\text { expectations }\end{array}$ & $\begin{array}{l}\Leftarrow \text { Integration } \Rightarrow \\
\text { of experience } \\
\text { information into } \\
\text { some } \\
\text { representation }\end{array}$ & $\begin{array}{l}\Leftarrow \text { Integration } \Rightarrow \\
\text { of new } \\
\text { advertising } \\
\text { information into } \\
\text { an associated } \\
\text { representation }\end{array}$ & $\begin{array}{l}\text { Regeneration } \Rightarrow \\
\text { of the altered } \\
\text { representation }\end{array}$ & $\begin{array}{l}\text { Response used in } \\
\text { decision making }\end{array}$ \\
\hline
\end{tabular}

SOURCE._-Adapted from Loftus (1975).

\section{Reconstructive Retrieval}

The focus of this investigation is given to the right-hand side of this diagram: reconstructive retrieval. Consumer experiences previously have been conceptualized by marketers as "patterns permanently retained in the central nervous system"' (Burke and Srull 1988, p. 56). New research suggests otherwise: "A keypoint of agreement between cognitive and biological theories is that memories do not preserve a literal representation of the world; memories are constructed from fragments that are distributed across different brain regions, and depend on influences operating in the present as well as the past" (Schacter 1996a, p. 13527). Research shows that when people try to recall a past experience, the information is assimilated within the existing memory schema, causing them to remember only what fits their expectations. At the same time, information acquired after an experience can transform the memory of that experience (Loftus 1982). In recall and recognition people tend to include ideas or elements inferred or related to the original experience but not actually part of the original experience itself (Bartlett 1932).

Postexperience information is most likely to distort memories when it is very similar to, or conjures up images which may be mistaken for, the actual experienced information. Garry et al. (1996) in their work on "imagination inflation" propose that recently generated images become accessible in the mind, people forget the source of their imaging, and they report the newly imagined event as having happened to them. Recent evidence on "verbal overshadowing" shows postexperience imaging can influence perceptual memories such as taste. Melcher and Schooler (1996) found that people who verbalize after tasting a wine show far lower accuracy in recognizing that wine than those that do not verbalize their experience. They believe that the recently generated mental representation overshadows what is learned in the perceptual experience because language cannot capture the complexity of the experience.

\section{Memory Reconstruction Hypothesis}

The present research proposes that marketers can manage consumers' reconstruction process through postexperience advertising messages that overshadow information learned in a direct experience. Postexperience advertising can create an association to the original experience by referencing sensory aspects and inducing positive imagery of that experience (Puto and Wells 1988). In the case of a direct taste experience, the representations elicited by the recently received postexperience advertising will overshadow respondents' memory of the actual taste so their memory will be of a more flavorful juice than they actually experienced. (One could conceive of this situation as a classic source-monitoring error where the mental representation of the taste becomes confused with the actual taste.) The memory created by the advertising will feel real to respondents, as real as a veridical memory. The memory effects will endure over time as brand schema information because own-experienced information is thought to be less resistant to decay (Neisser 1982).

\section{EXPERIMENTAL OVERVIEW AND ALTERNATIVE EXPLANATIONS}

The purpose of this experimental investigation was to determine whether objective sensory information such as taste can be transformed in memory through exposure to postexperience advertising and, if so, to determine whether this occurs prior to judgment processes. In other words, do consumers know they are being influenced in this manner? The misinformation paradigm from cognitive psychology has been adopted to investigate these issues. In this paradigm respondents experience an event; in the present case, taste a new orange juice. Later some respondents receive suggestive information, here advertising suggesting the taste was flavorful, while a control group does not receive this information. All are asked to identify what they experienced earlier (e.g., the orange 
juice they tasted). The respondents' tendency to accept information presented after the direct experience as being true of the original experience, that is, identifying a more flavorful juice than the one they had tasted, is evidence that the postexperience advertising systematically influenced the reconstruction process.

Several marketing theorists have found postexperience information has affected consumer judgments and have described these effects in terms of a belief updating process (Hoch and Ha 1986; Levin and Gaeth 1988; Smith $1993)$. Although learning new information may update beliefs, the cognitive effects of this process have not been investigated, and in some cases, researchers have inferred a memory process that is inconsistent with what we now know regarding the constructive nature of memory. Hoch and Ha (1986) suggest that the recently received information may momentarily inhibit retrieval of the experienced information, rather than change its composition. The other two articles used information integration theory to describe their effects; Levin and Gaeth (1988, p. 377) propose that "the two sources of information available to the judge are balanced in arriving at an integrated impression." Similar to the Bayesian independence assumption, information integration theory states information is discretely stored in memory and that attribute information is separate from judgment. It further proposes that there is no reorganization in memory (Anderson 1991). This independence assumption has been found to be limiting in explaining memory and inference effects (e.g., Huber and McCann 1982).

It is proposed here that postexperience advertising can have an effect on memory prior to the judgment process. Loftus et al. (1989) find that newly created memories from postexperience suggestion are reported quickly, with high confidence, countering the idea that two pieces of information are consciously compared in judgment. Moreover, researchers' efforts to reduce the postexperience effect on memory of their respondents by warning them about the misleading information's lack of credibility are not effective (Greene, Flynn, and Loftus 1982). Further, neurological evidence suggests this modification occurs prior to higher cortical processing (Schacter 1996b). As a means to further investigate the judgment issue, the quality levels of initial taste quality experience were varied in this experiment. One might suspect that as the discrepancy between advertising and experience got large (e.g., really bad taste and very positive advertising), there would be less reliance on the advertising if the latter information is weighted at the point of judgment (Smith 1993).

Missing from the judgment models is an explanation of why consumers believe their past experience had been as suggested by the ad. Consumers may falsely assess product experiences and overstate diagnosticity of those experiences if the advertising alters their memory (Hoch and Deighton 1989). The phenomenological aspects of remembering are important to understanding why consumers believe they are relying on their own past experi- ence but show evidence of advertising influencing their memory.

The subjective experience of remembering, of becoming consciously aware in the present of a past feeling or event, is itself an experience. "It is autonoetic consciousness that confers special phenomenological flavour to the remembering of past events, the flavour that distinguishes it from other kinds of awareness such as those characterizing, perceiving, thinking, imaging, or dreaming.' (Tulving 1985, p. 3 ). It is that type of experience that can make us believe the representations are veridical, unaffected by other information.

New measures are allowing us to capture how respondents feel about the product of their memory retrieval. According to Tulving (1985), the phenomenological aspects of remembering differ. "Remembering" is a conscious reexperiencing of the original event, while " knowing" is general knowledge learned without consciously accessing the past. Tulving (1985) found that by asking respondents about the quality of their memory, using this "remember" versus "know" distinction (R/K) when they recall or recognize a previously encountered item, the probability of the "remember" response can serve as an index of the extent to which autonoetic (explicit) consciousness is involved in the recovery of past events. This R/K measure has been validated on the human brain using positron emission tomography (PET). Researchers find that "remember' judgments produce greater metabolic activity in the sensory cortices (Schacter, personal communication, 1997).

The experimental section is organized as follows: first, a pilot study is described where the taste stimuli and memory test are calibrated. Experiment 1 tests whether postexperience advertising influences consumer memory of a taste experience. After finding a memory effect, experiment 2 addresses additional concerns regarding the fate of these experiential memories over time and in more typical consumer contexts. In this experiment, the advertising was found to exert a deep structural change in memory, particularly if the information had been consistent with the initial experience. It was hypothesized that the postexperience advertising information exerted two levels of effect: the first in the immediate verbal overshadowing found in experiment 1 across all levels of quality, and the second in the development of a brand schema found in experiment 2 . A follow-up study was conducted to determine whether the verbal overshadowing effect could be reinstated after a week's delay by providing the advertising closely before recall.

\section{PILOT TEST}

A pilot test was conducted to ensure that the three levels of orange juice used in the main experiments were perceived as qualitatively different. Respondents rated each of the three orange juice samples on seven-point attribute scales: taste; sweetness; consistency; freshness; 
ingredients; and quality. ${ }^{1}$ These attributes loaded on one factor, with coefficient alpha of .90 , and were combined to form an average rating of evaluation. The three samples were found to be distinct, $F(2,216)=57.82, p<.0001$. Post hoc comparisons using the Bonferonni technique (set at $p<.05$ ) found each level to be significantly different from one another, $\bar{X}=2.3$ for the "bad" sample, $\bar{X}=3.3$ for the "medium" sample, and $\bar{X}=4.1$ for the "good" sample. Overall 71 percent properly identified the "'good" sample correctly as the best, and 89 percent properly identified the "bad" as the worst.

\section{EXPERIMENT 1}

Respondents received a sample of a "new" orange juice. The sample was either "good," "'medium," or "bad" tasting, depending upon the experimental condition. Later in the hour, half of the respondents were presented advertising information that suggested the orange juice had excellent taste quality. All respondents were then tested on the memories of their taste experiences. The primary issue was whether postexperience advertising would change how they remembered their taste experience; would they be more likely to recall and identify a more favorable taste experience because of the advertising information?

\section{Method}

\section{Participants}

One hundred fifty undergraduates ( 66 female, 84 male ) participated in this study for course credit.

\section{Design}

A 3 (level of experience: good, medium, bad) $\times 2$ (advertising: advertising, no advertising) between-subjects design was used. Respondents were assigned randomly to one of these six conditions. Any differences in cell sizes are due to respondents skipping over measures in the questionnaire (which did not occur often).

\section{Stimuli}

The three orange juice levels were those pretested in the pilot study. For the memory identification test, respondents were asked to pick the one from among five samples that matched the flavor they tasted earlier. The three aforementioned levels were used - the "bad" sample was labeled ' 2 ,', the "medium'" sample labeled ' 3 ,', and the "'good' sample labeled '4.', In addition, respondents had the choice of picking two other samples in their memory test: "best'" sample, labeled "5," made with the Minute Maid concentrate according to directions and a

\footnotetext{
${ }^{1}$ The samples were prepared according to a recipe developed by Pechmann and Ratneshwar (1992).
}

“"worst", sample, labeled " 1 ," having twice as much vinegar, water, and salt as the "bad" sample.

The identification test was modeled after McCloskey and Zaragoza's (1985) modified test procedure, which they say reduces the likelihood that the postexperience influence is occurring at the point of judgment. In their modified test, the choice is between the original accurate information and a new piece of information. In the present memory test respondents choose among a wide range of alternatives - five discrete orange juice taste formulations. These levels also allow for a blended representation to be elicited, where both the taste evidence and advertising participate in the reconstruction, forming a new representation (Loftus 1977; Metcalfe 1990). As in the modified test, respondents are asked to correctly identify what they tasted earlier - a sample of orange juice - from alternatives they previously had not tasted. This modality match is important because some psychologists (e.g., Pezdek and Greene 1993) have criticized verbal tests in misinformation experiments because they are a closer match to the postexperience information than the original event and may result in a bias toward that response if a familiarity criterion is adopted.

\section{Procedure}

Respondents came to the setting expecting an orange juice taste test. The experiments were run in groups ranging from 13 to 18 people. The experiment began with respondents tasting a sample of "Orange Grove" orange juice (a fictitious brand name). In order to reduce demand effects, the experimenter said that she was running the study for an outside company, and they should report only their true feelings because that is what will be most helpful to the manufacturer for the introduction of this new product ( such instructions have been used in other advertising/experience studies to create a disassociation; e.g., Smith and Swinyard 1983). Respondents were told that this was a new orange juice brand on the market and that they would later be asked to evaluate its taste. Depending upon the experimental condition, respondents received a two-ounce sample of either the "good," "medium," or "bad"' orange juice and ate crackers to cleanse their palates. They also inspected a mock-up "Orange Grove" container.

Respondents were told that they would be making judgments about the juice later in the hour but first needed to complete a survey for another instructor. This distraction task lasted about 15 minutes and had no relationship to the present experiment. The advertising groups were then told that Orange Grove was trying to determine the type of ad campaign that would best launch their product on a national level. Respondents were shown two ads to evaluate within a five-minute time period. Both ads were transformational in nature, describing the positive taste qualities of the Orange Grove juice. For instance, one ad touted the home-grown company origins and asked readers to "imagine the taste of fresh squeezed orange juice 
. . . it's sweet, pulpy and pure.' The other ad compared the packaging of Orange Grove (a picture of an orange) to the packaging of frozen concentrate juice, describing how Orange Grove preserves the "integrity of Florida orange taste", by cutting out the middleman. Both ads ended with the slogan, "Orange Grove. Experience the taste Florida's been talking about.' Respondents rated each ad on a number of scales and wrote down their thoughts about each ad.

All respondents then completed a five-minute distraction task which asked about their own orange juice consumption. Respondents were subsequently asked to recall in their own words their taste experience with Orange Grove. They were asked to pick three words that best described their memory of Orange Grove's taste.

Then respondents were given five samples of orange juice and were asked to identify the one they remember having tasted earlier in the hour. They were told that one among the five samples was exactly as they had tasted earlier. The samples were numbered 1 to 5 , with 1 being the worst tasting and 5 being the best tasting. Respondents were not told the meaning of this ordering. They were told to sample each in numeric order and to defer making an identification until all five had been sampled. ${ }^{2}$ They were offered crackers to cleanse their palate between each sample.

After they identified the sample they believed to be Orange Grove, respondents distinguished between states of awareness using the process developed by Tulving (1985). In addition, a guess option was offered for those respondents who had no memory of the orange juice's taste (in order to rule out a guessing bias in the recognition test). Respondents were told a "remember' judgment is the strongest type of memory judgment and to indicate so if their memory for the orange juice approached a reexperiencing of it, that is, if they could taste the juice in their mind's eye. They were told to indicate a memory judgment as a "know" if it was based more on recognition or familiarity and if somehow the taste seemed appropriate, whether or not they could recall the prior taste itself. And they were told to indicate a memory judgment as a "guess" if they randomly selected one of the five samples as the Orange Grove and they had no recollection of its taste.

The respondents then rated their confidence in memory identification and subsequently were told that the experimenter would be giving them 50 cents at the end of the experiment as a "thank you,', and that they had the option of betting this money on their identification memory. They could choose to keep the full 50 cents and not wager anything, they could bet all if they believed they had been correct, or bet values in between. Finally, respondents rated their overall evaluations of the Orange Grove juice.

\footnotetext{
${ }^{2}$ This was done so that any order bias would be controlled for.
}

\section{Results}

\section{Coding Procedure}

Two independent judges coded respondents' reactions to the ads and the recall statements of their taste experiences. They had no knowledge of the experimental hypotheses. Their interrater reliability was .99 and an average was used for the analysis.

\section{Manipulation Check}

It was predicted that the transformational wording of advertising would cause respondents to conjure up sensory aspects of Orange Grove's taste. Respondents rated and gave feedback on the two ad campaigns. The judges looked at this feedback for mentions of sensory aspects of the juice, whether respondents mentioned taste when asked what the ad made them "think about" and if respondents mentioned any autobiographical experiences with orange juice. Eighty-five percent of respondents mentioned taste or sensory aspects of the juice somewhere in these ad evaluations. For instance, one respondent in the "bad" orange juice condition said the ad made him think of " a juicy orange bursting in my mouth.' Seventytwo percent of respondents specifically mentioned orange "taste"' when asked directly, "What did the ad make you think about?" A common response was, "Eating fresh oranges."' Others directly mentioned the imagination process: one respondent in the "medium" taste condition wrote, "The best orange juice I could ever imagine." Fourteen percent of respondents conjured up their own past experiences when viewing the ads; one respondent in the "good" experience group said the ad made her think of "fresh squeezed orange juice like Mom used to make.' The judges found no difference in the number reporting sensory images or taste experiences across the quality levels, $F<1$. $^{3}$

\section{Recall}

Respondents had five minutes to describe in detail memory of their taste experience of Orange Grove. The judges coded respondents' free recalls for the number of words used in the description; the total number of independent thoughts; whether the thoughts seemed positive, negative, or neutral; and whether any words (or variants of words) that had appeared in the ads describing

\footnotetext{
${ }^{3}$ This was meant to be a manipulation check rather than a process tracing measure. It is very possible that those who did not write down thinking about the taste, conjured it up. During the ad viewing, respondents were not asked specifically to think about the taste of the orange juice but, rather, to rate the ads. The correlation between those who mentioned taste and subsequently reported a distorted memory was $r$ $=.26$. Of the 67 respondents that mentioned taste, 51 reported a taste identification other than accurate, with 40 choosing a more favorable taste match.
} 
TABLE 1

FREE RECALL ANALYSIS

\begin{tabular}{|c|c|c|c|c|c|c|c|c|c|}
\hline \multicolumn{2}{|c|}{ Group } & \multicolumn{5}{|c|}{ Describe taste in your own words } & \multicolumn{3}{|c|}{$\begin{array}{l}\text { Pick three words that best } \\
\text { describe taste (number) }\end{array}$} \\
\hline Ad & $\begin{array}{l}\text { Orange } \\
\text { juice }\end{array}$ & $\begin{array}{l}\text { Number } \\
\text { of words }\end{array}$ & $\begin{array}{l}\text { Number of } \\
\text { thoughts }\end{array}$ & $\begin{array}{l}\text { Positive } \\
\text { (\%) }\end{array}$ & $\begin{array}{l}\text { Negative } \\
\text { (\%) }\end{array}$ & $\begin{array}{l}\text { Number } \\
\text { of words } \\
\text { from ad }\end{array}$ & Positive & Negative & $\begin{array}{l}\text { Words } \\
\text { from ad }\end{array}$ \\
\hline \multicolumn{10}{|c|}{ Experiment 1: } \\
\hline Yes & Good & 29 & 3.78 & 57 & 20 & 2.57 & 1.8 & .59 & 1.32 \\
\hline No & Good & 33 & 3.3 & 31 & 48 & 1.82 & 1.15 & 1.36 & 1.3 \\
\hline Yes & Medium & 30 & 3.38 & 41 & 38 & 1.9 & 1.67 & .87 & 1.5 \\
\hline No & Medium & 33 & 3.9 & 33 & 59 & 1.67 & .70 & 1.73 & 1.35 \\
\hline Yes & Bad & 29 & 3.42 & 37 & 44 & 2.0 & 1.26 & 1.0 & 1.3 \\
\hline No & Bad & 25 & 3.31 & 27 & 59 & 1.62 & .75 & 1.5 & .81 \\
\hline \multicolumn{10}{|c|}{ Experiment 2: } \\
\hline Yes & Good & 22 & 3.19 & 54 & 38 & 2.3 & 1.74 & 1.05 & 1.54 \\
\hline No & Good & 22 & 3.01 & 35 & 50 & 1.48 & 1.2 & 1.38 & 1.21 \\
\hline Yes & Bad & 25 & 3.08 & 27 & 62 & 2.09 & .9 & 1.71 & 1.19 \\
\hline No & Bad & 22 & 3.63 & 17 & 72 & 2.23 & .51 & 2.22 & 1.25 \\
\hline \multicolumn{10}{|c|}{ Follow-Up: } \\
\hline Yes & Good & 17 & 2.7 & 62 & 23 & 2.36 & 1.79 & .27 & 1.79 \\
\hline Yes & Bad & 20 & 3.2 & 32 & 39 & 2.27 & 1.14 & 1.23 & 1.61 \\
\hline
\end{tabular}

Orange Grove's taste appeared in those descriptions. The results by condition appear in Table 1 .

There was no significant difference between conditions on the quantitative aspects of recall (e.g., number of words and thoughts ). The conditions differed in the qualitative aspects. The memory reconstruction hypothesis predicted that language in the advertising would influence how respondents would remember their own taste experiences and that respondents would be more likely to use words from the advertising in their memory descriptions and to express more positive thoughts. For the percent of positive thoughts, advertising was a significant factor, $F(1,149)=7.6, p=.006$; experience was not significant at $F(2,149)=1.79, p=.17$. The interaction was nonsignificant, $F<1$. The number of positive words used to describe the juice advertising was significant at $F(1,149)$ $=13.86, p=.001$; type of experience was significant at $F(2,149)=2.35, p=.09$ with the interaction, $F<1$. For percentage of negative thoughts, both factors were also significant: $F(1,149)=17.36, p<.001$ for advertising; $F(2,149)=5.01, p=.007$ for level of experience; again, the interaction was nonsignificant, $F<1$. For the number of negative words, only the advertising factor was significant, $F(1,149)=15.0, p<.001$.

As predicted, respondents were more likely to use the descriptive terms from the ad (compared to the control groups ) when describing their own taste experience, $F(1$, $149)=4.20, p=.04$, and in their choice of the three words that best describe the juice, $F(1,149)=2.66$, $p=.01$. Neither the type of experience nor the interaction were significant factors. To further investigate the role language played on respondents' taste memories, a comparison was done between those respondents who received the advertising and later identified a juice other than the one they tasted as being Orange Grove (memory
TABLE 2

TASTE RECOGNITION

\begin{tabular}{|c|c|c|c|c|c|c|}
\hline \multirow{2}{*}{$\begin{array}{l}\text { Sample } \\
\text { identified }\end{array}$} & \multicolumn{2}{|c|}{$\mathrm{Bad}$} & \multicolumn{2}{|c|}{ Medium } & \multicolumn{2}{|c|}{ Good } \\
\hline & No ad & Ad & No ad & Ad & No ad & Ad \\
\hline \multicolumn{7}{|c|}{ Experiment 1: } \\
\hline 1 & 5 & 3 & 0 & 0 & 0 & 0 \\
\hline 2 & $11^{\mathrm{a}}$ & $5^{\mathrm{a}}$ & 7 & 6 & 6 & 2 \\
\hline 3 & 6 & 6 & $10^{\mathrm{a}}$ & $8^{\mathrm{a}}$ & 6 & 6 \\
\hline 4 & 2 & 6 & 3 & 9 & $13^{\mathrm{a}}$ & $8^{a}$ \\
\hline 5 & 0 & 7 & 0 & 4 & 1 & 10 \\
\hline \multicolumn{7}{|c|}{ Experiment 2: } \\
\hline 1 & 5 & 0 & $\ldots$ & $\ldots$ & 1 & 0 \\
\hline 2 & $12^{\mathrm{a}}$ & $12^{\mathrm{a}}$ & $\ldots$ & $\ldots$ & 5 & 0 \\
\hline 3 & 7 & 11 & $\ldots$ & $\ldots$ & 7 & 4 \\
\hline 4 & 2 & 4 & $\ldots$ & $\ldots$ & $9^{a}$ & $11^{a}$ \\
\hline 5 & 0 & 2 & $\ldots$ & $\ldots$ & 6 & 11 \\
\hline \multicolumn{7}{|c|}{ Follow-Up: } \\
\hline 1 & $\ldots$ & 3 & $\ldots$ & $\ldots$ & $\ldots$ & 0 \\
\hline 2 & $\ldots$ & $1^{\mathrm{a}}$ & $\ldots$ & $\ldots$ & $\ldots$ & 0 \\
\hline 3 & $\ldots$ & 7 & $\ldots$ & $\ldots$ & $\ldots$ & 2 \\
\hline 4 & $\ldots$ & 4 & $\ldots$ & $\ldots$ & $\ldots$ & $8^{a}$ \\
\hline 5 & $\ldots$ & 7 & $\ldots$ & $\ldots$ & $\ldots$ & 15 \\
\hline
\end{tabular}

andicates correct identification.

distortion) to those who reported accurate identifications. The advertising language was significantly more likely to appear in those misled or random respondents' recall statements, $\bar{X}=2.24$ words, than for accurate respondents, $\bar{X}=1.3 ; F(1,78)=6.63, p=.01$.

\section{Memory Identification}

Respondents were asked to pick the sample that they had tasted earlier in the hour. The distributions for each condition appear in Table 2. 
Accuracy was higher when respondents did not receive the advertising information, demonstrating that the postexperience advertising information overshadowed the perceptual experience and impaired memory; accuracy for control was 46 percent, and for advertising, 26 percent; significantly different, $\chi^{2}(1, N=150)=6.190, p=.013$. More critical, the statistical analyses confirmed the power of the advertising information to positively transform the perceptual memories of consumers' taste experiences, as respondents were more likely to choose a more favorable sample to represent their original experience if they received the ads. Because the dependent variable-the choice of the orange juice-was ordinally scaled where 1 was the worst tasting and 5 was the best tasting, a categorical data analysis procedure was used to analyze the means of the recognition data. With this analysis, both the main effects (e.g., ad and level of experience) and their interaction could be incorporated into the model. Both advertising and level of experience were found to be significant factors: advertising $\chi^{2}(1, N=150)=27.64$, $p<.00001 ;$ and level of experience $\chi^{2}(2, N=150)$ $=23.19, p<.00001$; the interaction was nonsignificant, $\chi^{2}<2$.

\section{Type of Memory Judgments}

In order to demonstrate that these taste memories had become reconstructed and seemed real to the respondents, several measures were taken: $\mathrm{R} / \mathrm{K} / \mathrm{G}$ to get at the consciousness of the recollection; a confidence scale anchored from 0, "not at all sure correct," to 100, "extremely sure correct', ; and the bet, where they could bet anything from 0 to 50 cents on their memory identification. $^{4}$

For this first analysis, only respondents who received the advertising information were included. They were divided into two groups: those who reported "accurate", memories, and those who reported "misled" or "blended" memories. The latter group was defined as those respondents in the advertising conditions who chose a more favorable identification match for the Orange Grove (41 respondents). There were 20 respondents who reported correct memory judgments. It was expected that if those who had been misled by the advertising did so because they thought their experiences ambiguous and were reporting based on confusion rather than a belief that their taste experience was as identified in the recognition test, then they would report lower confidence, less "'remember', judgments, and would bet less than their accurate counterparts. This was not the case. There were no differences in confidence, $\bar{X}=67$ for misled, $\bar{X}=68$ for accurate; no difference in percentage of "remember",

\footnotetext{
${ }^{4}$ As Donaldson (1996) found, confidence and $\mathrm{R} / \mathrm{K}$ was correlated, $r$ $=.49$, but not so high that they were measuring the same thing. The bet was not highly correlated with either confidence, $r=.18$, or the R/ $\mathrm{K}$ measure, $r=.10$, so it may have been measuring something else, like risk taking.
}

judgments, 40 percent for misled, 38 percent for accurate; and no difference in amount bet, 36 cents for accurate versus 40 cents for misled. ${ }^{5}$

One might argue that those who received the advertising information had more information and thus would be more confident in their decisions (e.g., knowingly updated their beliefs). Differences between those who received the ad information and those who did not were also examined, but there was no difference: $\bar{X}=66$ for confidence for those who received the ad, $\bar{X}=64$ for noad groups; $\bar{X}=37$ cents bet for ad groups, $\bar{X}=36$ cents bet for no-ad groups; and 36 percent 'remember' for ad groups, 37 percent for no-ad groups. In short, there was no objective manner to differentiate an "accurate", memory from a "misled" one created by the advertising.

\section{Overall Evaluations}

The interest was in whether or not these changes in sensory memories would translate to overall evaluations of the juice. The seven subjective measures loaded on one factor, with coefficient alpha of .95 . They were combined to form an index of subjective evaluation. Advertising was a significant factor, $F(1,149)=21.45$, $p=.0001$; the level of experience was significant at $F(2$, $149)=11.8, p=.0001$. The interaction was nonsignificant, $F<1$, therefore assimilation of the postexperience advertising occurred at all levels of experience.

\section{Discussion}

The purpose of this study was to demonstrate that postexperience advertising could make consumers think that they had tasted a more flavorful juice by altering their memories of the tasting experience. The statistical analyses confirmed the power of suggestive advertising to transform memories, but the respondents' verbatim words more clearly demonstrate the impact. For instance, one respondent who had tasted the vinegar-tinged salty orange water and received the ad information described his memory of the orange juice's taste in these words, "I thought it tasted real sweet. It quenched my thirst. Refreshing. It would be a nice eye-opener in the morning. It made me want more." Another respondent who tasted the same juice but who did not receive the advertising information described his memory as, "I thought this juice was pretty

\footnotetext{
${ }^{5}$ For the purposes of this investigation null results are interesting because, based on current theory, we might expect great differences, and for the purpose of the present investigation they were not found. Of course, at some level a difference, no matter how minute, can be found statistically different. Power depends on sample size (within groups ), effect size, the number of groups, and the standard error; where the noncentrality parameter $\phi=\sqrt{ }\left(\eta_{j} \alpha^{2}\right) /(a \sigma)$, where $\eta_{j}$ is the number within each group, $\alpha$ is the treatment effect, $a$ is the number of groups and $\sigma$ is the standard error (as per Feldt [1993], p. 40). With the present standard deviations and sample size, a treatment effect of 13 for confidence, 30 percent for remember, and 10 for amount be would have been needed to achieve a power of .75 .
} 
terrible. It was bitter and watered-down." ${ }^{6}$ Though both respondents experienced the same bad juice, this experiment demonstrates that these experiences can be malleable ex post facto at both objective and affective levels, and what the consumer ultimately comes to remember may differ significantly from the initial representation, depending on what information s/he receives afterward.

The alternative explanation to these memory changes is that respondents knowingly updated their beliefs about the product at the point of judgment. This is consistent with Norman Anderson's information-integration theory. He specifically rejects Solomon Asch's conjecture of a gestalt-memory representation where impressions are formed through a complex interaction of attributes (Anderson 1991). Information-integration theory assumes verbal memory is distinct from other attitudinal information, and the interaction or cross-modal influence of advertising should not effect the sensory representation of the experience itself. In information integration, the newer advertising information should be added on to the judgment; with a reconstructive view there is a constant reorganization in memory. Steps were taken to differentiate between a change in memory and a process occurring solely as a conscious judgment. In their critique of memory-distortion findings McCloskey and Zaragoza (1985) posed two alternative routes that could produce outcomes that appear to be memory changes but actually occur in judgment: vacant slot and coexistence. These explanations will be introduced, and evidence from the experiment will be presented which makes these routes appear unlikely, thus leaving us with the memory reconstruction explanation. A "vacant slot" explanation would propose that respondents may not have encoded the original experience, accepted the postexperience information, and knowingly used it to fill in the missing information with that newer information. The memory itself never changed because it was never well encoded. In visual events this notion, known as vacant slot, may be more likely to occur when information was peripheral to the events. In the present study, this is unlikely because the target information is central to the experience; respondents directly tasted the juice and millions of taste and olfactory sensory receptors encoded this information (Cytowic 1993). Further, respondents were told that they would be making judgments about the orange juice at a later time to ensure they would form some impressions of its flavor. Had guessing been evident, showing no memory of taste, we might have predicted a more uniform distribution in the recognition test in the control conditions rather than distributed around the accurate response. A "coexistence", explanation would propose that respondents might have both pieces of information in memory and opt for the most recent information due to other reasons, such as

\footnotetext{
${ }^{6}$ There were several more negative statements than the one presented in the article, but I thought they would not be appropriate to share in an academic journal.
}

demand effects or recency in presentation. This is similar to the argument made by Hoch and Ha (1986) who state that the recently received information inhibits rather than changes recall of the actual experience. The recognition test helped rule out this possibility. The modality match forces respondents to consider their original taste experience rather than the more recently received advertising. Picking a sample other than the one they tasted (and holding it with high confidence) constitutes a perceptual change in memory (Pezdek and Greene 1993). Experiment 2 investigates the possibility of demand.

The $\mathrm{R} / \mathrm{K}$ measures further provide some insight into how these newly created memories feel to the rememberer. Tulving (1985) says the phenomenon of remembering is determined jointly by semantic and episodic information. Respondents' taste memories were rich, involving affective and sensory components. Many respondents reported remember-type judgments where they could "taste the orange juice in their mind's eye." And as Schacter (1996b) notes, that feeling associated with remembering of reexperiencing the past can trick us into believing in the accuracy of those recollections. What they were reexperiencing in this case wasn't driven by their actual product taste experience but, rather, by the images and words evoked in the advertising.

This kind of reexperiencing was predicted by the memory-reconstruction hypothesis. Perhaps advertisers cue consumers to imagine favorable experiences and thus explicitly alter how they remember past product encounters. Memory researchers find that both language and imagery can help people remember accurate information and distort veridical memories. "Of the sources of memory illusions our own language may be the most insidious. We depend on language to represent our experiences faithfully so that we can communicate them to others and so that we can remember them ourselves" (Melcher and Schooler 1996, p. 231). Advertising's ability to provide the language that guides how consumers imagine past experiences may therefore affect postexperience choice decisions. This finding is related to recent research by West, Brown, and Hoch (1996), who found that external information can serve as a consumption vocabulary that helps consumers understand and develop their own preferences.

The memory reconstruction process is influenced by two types of information: schemas and environmental cues. In the present experiment there was little or no prior brand information that would contribute to the Orange Grove schema, only prior category knowledge, which would allow respondents the background to differentiate between a "good" and "bad" juice. Because the postexperience advertising was presented relatively close in time to the retrieval process, it might have been part of the environmental cuing process that led to the newly created memories of the juice. The verbal overshadowing effects have been momentary where the recently generated image is used at the expense of the actual perceptual experience (Melcher and Schooler 1996). Whether or not this infor- 
mation becomes incorporated into consumers' brand schema needs to be investigated over time.

\section{EXPERIMENT 2}

This investigation is divided into two parts that study the postexperience advertising effects a week after the information was received. Part A looks at whether the postexperience advertising would have enduring effects on more typical consumer tasks such as consideration sets and choices. Research by Johnson and Seifert (1994) indicates that the misinformation (or suggestion) becomes incorporated into consumer associations and affects later inference making and judgments. Anderson's meaning-constancy hypothesis says that informational inputs such as the orange juice taste have a fixed meaning that is used in forming a judgment, and any effects of the advertising information (or other contextual information) occur subsequent to this process. According to information-integration theory, such context changes may be illusory and disappear in new contexts (Anderson 1991; Lynch, Chakravarti, and Mitra 1991). Part B looks at the state of the taste memories over time. Would the effect of postexperience advertising disappear because it was no longer more accessible than the actual experience? Or would the postexperience advertising continue to exert an effect on the reconstruction of the experience? Rather than exerting an influence on the environmental cues at retrieval, the influence comes from becoming integrated into Orange Grove's brand schema.

\section{Method}

\section{Participants}

One hundred eleven undergraduates ( 66 female, 45 male) participated in this study for course credit. The final analysis includes 106 respondents because five respondents failed to appear for the second week (these were equally distributed across experimental conditions ).

\section{Design}

A 2 (level of experience: good, bad) $\times 2$ (advertising: advertising, no advertising) between-subjects design was used. Respondents were randomly assigned to one of these four conditions.

\section{Procedure}

The same basic procedure for experiment 1 was followed at week 1, when respondents tasted the orange juice (good/bad) and then half later received the advertising information. All respondents filled out background information about their orange juice consumption, were thanked for participating in the taste test, and left with the impression that the orange juice experiment was complete.

\section{Part A}

A different experimenter came in and said she was doing some exploratory research on Internet usage and how it affects other media behaviors. She requested that they return the following week and report on their media usage during the week. Questions about brands were included in her subsequent survey of generation X. Among the questions were those of a general nature relating to types of brands people their age consider and choose when making decisions. There were three levels of interest: evoked sets, consideration sets, and choice decisions. Their operationalizations appear below. Respondents completed these questions first with soft drinks for practice, then for the target orange juice, then for candy bars.

Evoked Sets. When you think of "orange juice", what brands come immediately to mind? Remember that these are brands that define the "orange juice" category for you, although they may not be the ones you would consider buying. Write down any (and all) brands that come to mind.

Consideration Sets. Which of the following brands would you consider buying if you saw them in the store? Remember, these are the brands you think are good enough to buy, and if priced right (or if your "favorite", were not available), would be an acceptable substitute. Place an ' $X$ ' in front of those brands that meet these criteria. Mark as many as apply. If there are additional brands that were not mentioned, list in the "other" category. (Orange Grove appeared second on the list of 17 brands.)

Choices. Below are sets containing two brands, and your job is to choose which one you would buy if they were the only ones available at the store, and you really wanted or needed orange juice. Place an ' $\mathrm{X}$ ' in front of the brand you would choose. Do this for each line (choice set). Buried within five other choice sets were the following three sets of interest:

Orange Grove or $\quad$ Hy-Vee
President's Choice or $\quad$ Orange Grove
Minute Maid or___ Orange Grove

\section{Part B}

After the generation $\mathrm{X}$ survey respondents participated in a short distracter task, they then completed the same memory measures that had been given in experiment 1 . To further investigate the possibility of demand, respondents were given the opportunity to guess the experimental hypotheses at the end of the study.

\section{Results}

Evoked Sets. The average evoked set size was 2.7. There was no difference in set size across experimental conditions. As expected, Minute Maid was listed most 
frequently, mentioned by 89 percent of respondents. HyVee, the local store brand, was mentioned by 17 percent of respondents. President's Choice was not mentioned by any respondents, nor was Orange Grove. Because Orange Grove was represented as a new brand and not yet nationally distributed, it was not expected that Orange Grove would be chosen as a defining brand in the juice category.

Consideration Sets. The consideration set size did not differ significantly across conditions, overall $\bar{X}=7.24$. Orange Grove was indicated as part of the consideration set more often when the advertising had been received, marginally significant at $F(1,105)=2.70, p=.1$; and when there had been a favorable taste experience, $F(1$, $105)=9.43, p=.003$. Specifically, Orange Grove was mentioned by 50 percent of the good experience/ad condition; 35 percent of the good experience/no-ad condition; 27 percent of the bad experience/ad condition; and 12 percent of the bad experience/no-ad condition. President's Choice was in 10 percent of the consideration sets; $\mathrm{Hy}-\mathrm{Vee}$ in 55 percent of the sets; and Minute Maid in 96 percent of the sets. There were no differences across conditions for these other brands.

Choices. The postexperience advertising had an influence on actual choices. For the choice between Orange Grove and President's Choice (an unknown alternative) advertising was significant at $F(1,105)=8.92, p=.003$; marginally significant for level of experience, $F(1,105)$ $=3.37, p=.06$. Specifically, 92 percent of respondents in the good experience/ad condition chose Orange Grove, 75 percent in the good experience/no-ad condition; 85 percent of in the bad experience/ad condition, and 54 percent in the bad experience/no-ad condition. For the choice between Orange Grove and Hy-Vee, a known local alternative, advertising was marginally significant, $F(1$, $105)=3.3, p=.07$; level of experience was significant at $F(1,105)=9.43, p=.003$. Seventy-three percent in the good experience/ad condition chose Orange Grove over Hy-Vee; 54 percent in the good experience/no-ad condition; 42 percent in the bad experience/ad condition; and 27 percent in the bad experience/no-ad condition. For the choice between Orange Grove and a well-known national, superior-quality brand, only advertising was a significant factor, $F(1,105)=5.55, p=.02$. Twelve percent of respondents in the good experience ad condition and 8 percent in the bad experience/ad condition chose Orange Grove over Minute Maid. No one in the no-ad conditions chose Orange Grove over Minute Maid.

\section{Coding and Manipulation Check for Part B}

As in experiment 1 , two independent judges coded respondents' reactions to the ads and the recall of their taste experiences. Their interrater reliability was .99 , and an average was used for the analysis. As observed in experiment 1 , the judges found that 84 percent of the respondents mentioned taste or sensory aspects of the orange juice somewhere in their response to the advertising; 77 percent specifically mentioned "taste" when asked directly, "What did the ad make you think about?" Thirteen percent conjured up their own past experiences when viewing the ads. ${ }^{7}$

\section{Recall}

The free recall results by condition appear in the middle portion of Table 1. As found in experiment 1, there was no significant difference between conditions on the quantitative aspects of recall (e.g., number of words and thoughts). It was the qualitative aspects of recall that differed ( $p$ values are reported as one-tailed $F$ for the following tests). For the percentage of positive thoughts, advertising was a significant factor, $F(1,105)=4.6$, $p=.02$; experience was also significant, $F(1,105)$ $=10.68, p<.001$; the interaction was nonsignificant, $F$ $<1$. The number of positive words used to describe the juice also followed this pattern: advertising was significant with $F(1,105)=4.45, p=.015$; level of experience, $F(1,105)=13.81, p=.001$; with the interaction, $F<1$. For percentage of negative thoughts both factors were again significant: $F(1,105)=1.7, p=.08$ for advertising; $F(1,105)=9.91, p=.007$ for level of experience; the interaction was nonsignificant, $F<1$. For the number of negative words both advertising and experience were significant factors: $F(1,105)=3.38, p=.03$ for advertising; $F(1,105)=12.18 p<.001$ for level of experience; interaction was nonsignificant, $F<1$.

In experiment 1 , respondents in the ad conditions were more likely than the no-ad groups to use descriptive words from the advertising in their recall in the same fashion across levels of experiences. Here, however, this was more likely to happen for the good taste condition, suggesting that words consistent with the schema are used in the reconstruction and endure as part of the brand experience. The interaction was significant, $F(1,105)$ $=3.96, p=.04$, with a main effect of advertising, marginally significant at $F(1,105)=2.25, p=.07$. There were no significant effects for the three words chosen to describe Orange Grove. In experiment 1, it was also shown that those who reported misled or random identification memories were more likely to use words from the ad in their recall statements than those who reported accurate memories. This was not the case here, however: there was no difference between accurate, $\bar{X}=2.05$, and misled, $\bar{X}=2.06$. (Note: the difference is not found because of less ad words by misled respondents but rather because of more ad words by the accurate respondents, suggesting perhaps the accurate respondents suppressed these words when the experience was more accessible in experiment 1 , but the words infiltrated memory over time.) As

\footnotetext{
${ }^{7}$ Again, this was meant to be a manipulation check, not a predictor of memory: there was no relationship between taste being mentioned at week 1 and distortion, $r=.01$. Forty-seven respondents mentioned taste: of those, 32 identified a juice other than the accurate one, with 25 identifying a more favorable juice.
} 
Melcher and Schooler (1996) note, language may be used to help stabilize "correct" memories as well as to overshadow the original perception.

\section{Recognition}

Recall that respondents were asked to pick which sample they had tasted the previous week. The distributions for each level appear in Table 2. Unlike experiment 1, there was no difference in accuracy between those who did and did not receive the advertising information, with 38 percent accuracy in both cases. However, as in experiment 1 , those who received the postexperience advertising were also more likely than their no-advertising counterparts to identify a more favorable juice as being Orange Grove. Both advertising and experience were significant factors: ad $\chi^{2}(1, N=106)=18.35, p<.00001$; level of experience $\chi^{2}(1, N=106)=54.1,7 p<.00001$; the interaction was nonsignificant, $\chi^{2}<1$. By looking at the table, it appears that the no-ad distributions seem more disperse than the ad conditions. These distributions were compared using the Empirical Distribution Function (EDF) analyses for nonparametric data and the Kolmogorov-Smirnov test found that there was more dispersion in the no-ad groups, marginally significant (KolmogorovSmirnov test statistic $=1.3, p=.06$ ).

\section{Type of Memory Judgment}

As in experiment 1, first the accurate and misled respondents in the advertising groups were investigated for qualitative differences in recall, $(N=20$ accurate, $N=$ 28 misled). Again, there were no significant differences in the consciousness of recall between those that had been misled and those that had reported accurate judgments; 45 percent ' 'remember' for accurate, 46 percent for misled; confidence, $\bar{X}=62$ for accurate, $\bar{X}=64$ for misled; or willingness to bet, $\bar{X}=34$ cents for accurate, $\bar{X}=37$ cents for misled. There was also no difference between the ad/no-ad conditions on confidence, $\bar{X}=63$ for ad groups, $\bar{X}=62$ for no-ad groups; or amount bet, 36 cents in ad groups, 40 cents in no-ad groups. There were more "remember' judgments in the ad groups, 47 percent versus 37 percent, though this did not reach statistical significance. $^{8}$

\section{Overall Evaluations}

As in experiment 1, the seven subjective measures loaded on one factor, with coefficient alpha $=.95$, and were combined to form an index of subjective evaluation. Both advertising and level of experience were significant factors, $F(1,105)=7.27, p=.004$ for advertising; $F(1$,

\footnotetext{
${ }^{8}$ The correlations between $\mathrm{R} / \mathrm{K} / \mathrm{G}$ followed the same patterns for experiments 2 and the follow-up study. Because similar patterns of the postexperience effects occurred on memory, it is highly unlikely these effects can be explained away due to lack of statistical power.
}

$105)=31.07, p<.001$ for type of experience. The interaction was nonsignificant, $F<1$.

\section{Demand}

No one reported that they thought the purpose of this study was to see if advertising could alter rememberedtaste quality of the juice.

\section{Discussion}

This experiment served several purposes. Part A was designed to see whether the memory effects found in experiment 1 would replicate in a more realistic consumer decision-making context or whether the previously found effects might be due to experimental demand. The results here suggest that postexperience advertising can have enduring effects on memory by altering respondents' subsequent choices. It also helps rule out a demand interpretation to experiment 1 because advertising's influence was found with another independent experimenter (Schwarz 1996).

Part B was designed to investigate consumer memories of taste experience over time to determine whether the findings of experiment 1 could be attributed to a momentary context effect rather than a true change in memory. The taste memories continued to be influenced by the postexperience advertising, albeit not in the same manner as observed in experiment 1 . In experiment 1 it was found that the recently presented advertising diminished identification accuracy and that those who used words from the ads within their recall statements also were more misled. In experiment 2 it was found that accuracy for both the advertising and control conditions had diminished but that the advertising continued to systematically affect recognition.

Recall that respondents had seen two ads, one that featured a picture of an orange, the other a historical description of the fresh-squeezing process. These images were still invoked in respondents' recall a week after presentation. This was especially true when the experience and advertising were somewhat consistent. For instance, according to one respondent who tasted the good orange (with the descriptive words from the ad appearing in italics): "The true taste of fresh-squeezed oranges. The juice tasted like real oranges.' Key words that advertising uses to evoke an experience may persist as part of the brand schema (such as "fresh-squeezed") and influence how the memory becomes reconstructed. Bartlett's schematic view of memory provides a theoretical explanation of considerable generality as to why experiences may be reconstructed in a manner that is incomplete or distorted. It also makes specific predictions regarding what should be used in the reconstruction process: relevant, typical, or consistent information (Alba and Hasher 1983 ). Within marketing, these results are consistent with schema theorists (Stayman, Alden, and Smith 1992) who find that information becomes incorporated into the repre- 
sentation when it is consistent with assumptions and can shape future expectations.

Ulric Neisser (1967, p. 285) used an analogy from Donald Hebb who likened the rememberer to a paleontologist who attempts reconstruction of a dinosaur from fragmentary fossil remains: "Out of a few stored bone chips we remember a dinosaur.', The advertising conditions had more fragments to use for their reconstruction of the orange juice taste. For those without such postexperience advertising support, identification of the original taste got more disperse with less "remember' judgments.

Perhaps the most notable difference between this experiment and experiment 1 is in the negativity for those who received the bad juice, especially if no advertising was received. In order to determine the effect of delay on memories, several comparisons were made to the groups of experiment $1 .^{9}$ The first comparison was with the recall statements, specifically those who tasted the bad juice. Both time and advertising were significant factors; with the advertising there were more favorable thoughts, marginally significant at $F(1,102)=2.73, p=.1$ for percent favorable thoughts and $F(1,102)=3.33, p=.07$ for percent negative ones; and over time there were more negative thoughts, $F(1,102)=5.72, p=.02$ for percent negative thoughts and marginally significant, $F(1,102)$ $=2.72, p=.1$, for percent positive thoughts. There were no other significant differences between the recall statements. A similar pattern emerged in the overall evaluations. Although both the good orange juice groups had evaluations very similar to those in experiment $1(\bar{X}=68$ for good juice/ad, $\bar{X}=49$ good juice/no-ad group in experiment $1 ; \bar{X}=62$ good juice $/$ ad, and $\bar{X}=49$ good juice/no-ad group), the bad juice evaluation seemed to grow more negative, especially for the bad/no-ad group (the means had been $\bar{X}=46$ bad juice/ad and $\bar{X}=34$ bad juice/no-ad group in experiment 1, compared to $\bar{X}=37$ bad juice/ad and $\bar{X}=26$ bad juice/no-ad group for experiment 2 ). Using the index as the dependent variable and time and advertising as the independent variables, the interaction was significant, $F(1,102)=4.05$, $p=.04$, with the bad/no-ad group significantly lower.

Can marketers reverse that negative trend by presenting the postexperience advertising a week after the experience

\footnotetext{
${ }^{9}$ In order to ensure the groups were similar on orange juice preferences, several measures were taken within one of the distraction tasks. Respondents were asked to indicate how often they buy orange juice (overall $\bar{X}=4.4$ for experiment $1, \bar{X}=4.3$ for experiment 2 , and $\bar{X}=$ 4.2 for the follow-up study, on a $1-6$ scale with 6 being "very frequently"'); how knowledgeable they are regarding orange juice $(\bar{X}=$ 4.1 for experiment $1, \bar{X}=4.1$ for experiment $2, \bar{X}=4.2$ for the followup, also on a $1-6$ scale with 6 being "very knowledgeable") ); whether or not they have ever had fresh-squeezed orange juice $(\bar{X}=82$ percent had in experiment $1 ; \bar{X}=86$ percent in experiment 2 ; and $\bar{X}=85$ percent for the follow-up); whether or not they liked orange juice $(\bar{X}$ $=93$ percent for experiment $1, \bar{X}=92$ percent for experiment 2 ; and $\bar{X}=89$ percent for the follow-up). There were no significant differences across studies on any of these measures, $F<1$ in all cases. In addition, this analysis was also done within the cells of each study, and again no differences were found.
}

and just before recall? This is investigated in the followup study.

\section{FOLLOW-UP}

The purpose of this follow-up was to determine whether advertising presented a week after the taste experience would lead to results similar to those found in experiment 1 , in which the advertising was presented before recall, or if a week's delay would be too late for influencing a memory of a past experience. ${ }^{10}$ To effectively distort experiential memories, advertisers might need to position advertising close in time to the consumer's experience, as per the encoding-specificity hypothesis (Thomson and Tulving 1970). Lindsay (1993) finds such source confusions are more likely when the event and the postexperience suggestion give rise to highly similar memory records. In addition, if respondents form a judgment about the experience at week 1, researchers find that such judgments are less susceptible to interfering information (Lynch and Srull 1982). Alternatively, advertising might have an even greater effect on memory reconstruction after the to-be-remembered experience has had time to fade (Loftus 1979; Payne, Toglia, and Anastasi 1994).

\section{Method}

\section{Participants}

Forty-seven undergraduates ( 23 female, 24 male) participated in this follow-up study for course credit.

\section{Design and Procedure}

Respondents tasted the (good/bad) orange juice at week 1 and received the advertising a week later just before recall (as in experiment 1).

\footnotetext{
${ }^{10} \mathrm{~A}$ reviewer noted that it was inappropriate to make comparisons across experiments. This concern is noted, and future research should consider doing a full experiment that incorporates delay. Efforts were made to ensure that testing conditions and respondents were similar across these experiments, however. The same presenters were used across the experiments. The sessions were run in the same building. The experiments were all conducted within the first two weeks of the semester to ensure motivated respondents, and the subject pool from which these respondents came represented similar demographic characteristics. Respondents in the later studies (experiment 2 and the followup) were asked whether or not they had heard anything about this experiment prior to their participation in it. None had. The same taste stimuli and advertising were used across all conditions. In addition, the background knowledge measures regarding orange juice showed no significant difference across studies. Because the basic cognitive process studied within these experiments is unlikely to vary from semester to semester, and the effect of delay is important for learning about advertising's influence on memory, these comparisons were included.
} 


\section{Results}

\section{Coding and Manipulation Check}

The same coding procedures described earlier were followed. The judges' interrater reliability was .95 , and an average was used for the analysis. As found in the previous experiments, 90 percent of the respondents noted the sensory aspects of the juice while reading the ad, 63 percent specifically noted taste when asked what the ad made them think about, and 15 percent mentioned past experiences with orange juice.

\section{Recall}

The free recall results by condition appear in the lower part of Table 1. The first comparison of interest is to the control condition of experiment 2 to demonstrate advertising's effect even after a week's delay. There were more positive thoughts when respondents received the advertising at recall, $F(1,99)=13.65, p<.001$, and had a good taste, $F(1,99)=13.95, p<.001$; less negative thoughts with the advertising, $F(1,99)=21.7, p<.001$, and the good taste, $F(1,99)=7.77, p=.006$; and more ad terms used in recall regardless of type of experience, $F(1,99)$ $=4.81, p=.03$, with the interaction nonsignificant. There were so few accurate responses $(N=9)$ that the comparison between the accurate and misled respondents was unwarranted.

The next comparison was between the advertising conditions of experiments 1 and 2. As noted in experiment 2 , there was a negative shift when the advertising had a week to decay, resulting in more negative thoughts and words; a difference across those conditions was significant at $F(1,146)=5.88, p=.003$. The advertising presentation in this follow-up study showed a reversal of this negative trend; post hoc comparisons ( set at $p<.05$ ) found that in experiment 1 and this study there were significantly fewer negative thoughts than in experiment 2 but not significantly different than each other. The negative words used to describe the juice also differed significantly across conditions, $F(1,144)=7.07, p=.001$, and post hoc comparisons revealed the same pattern where there were fewer negative words used in this follow-up than in experiment 2 but not significantly different than in experiment 1.

\section{Recognition}

As in experiment 1, there was a difference in accuracy between those who did and did not receive the advertising information: 38 percent of respondents in the control groups, 17 percent from the ad groups, which is significantly different at $\chi^{2}(1, N=101)=5.02, p=.03$. This accuracy rate in the ad groups was not significantly different from that of experiment $1\left(\chi^{2}<1\right)$ but was less than experiment $2\left(\chi^{2}(1, N=97)=4.76, p=.03\right)$, indicating that memory impairment returns when the ad is presented close in time to the memory test. As found in both experiments 1 and 2, there was a systematic shift in recognition based on the advertising, where those that received the advertising identified a more favorable sample than did the control group, $\chi^{2}(1, N=101)=30.09$, $p<.00001$. The postexperience advertising exerted more impact on recognition when it occurred right before the test than in experiment 2 when it had a week to fade, $\chi^{2}$ $(N=96)=2.48, p=.05$; and was also more influential than experiment 1 when the experience had been more accessible, marginally significant at $\chi^{2}(N=99)=2.31$, $p=.06$.

\section{Quality of Memories}

The created memories formed through postexperience advertising were held with high confidence, $\bar{X}=65$, and respondents were willing to bet on them, $\bar{X}=38$ cents. There were no significant differences in these measures between this and the previous experiments, $F<1$ in both cases. The phenomenological aspects did differ, however. Forty-three percent reported "remember" judgments in this experiment, which is marginally more significant than the 28 percent in experiment $1, \chi^{2}(N=100)=2.22$, $p=.06$, but not significantly different than the 47 percent in experiment 2 . There was no significant difference in the number of guesses across these postexperience advertising conditions, $\chi^{2}<1$.

\section{Overall Evaluations}

As in the other experiments, the subjective measures loaded on one factor with coefficient alpha $=.94$, and an index of evaluation was formed. Evaluations were higher with than without the advertising, $\bar{X}=67$ for good orange juice, $\bar{X}=45$ for the bad orange juice; significant from the control groups at $F(1,98)=15.17, p=.0002$ for advertising; $F(1,98)=25.01, p=.0001$ for level of experience; interaction, $F<1$. It was found in experiment 2 that postexperience advertising's effect on evaluations decreased over the week's time $(\bar{X}=61$ for the good orange juice compared to experiment $1, \bar{X}=68 ; \bar{X}=34$ for the bad orange juice, compared to $\bar{X}=45$ for experiment 1$)$. The advertising presented at the time for recall raised the evaluations, where the evaluations were higher than experiment 2 but not significantly different than experiment 1 . The postexperience advertising effect on evaluations was tested using ANOVA with time and level of experience the independent variables. Both time and level of experience were significant factors, $F(2,148)=2.91$, $p=.05$ for time with post hoc comparisons showing significant differences ( set at $p<.05$ ) between experiment 2 for both experiments 1 and $2 ; F(1,148)=40.62$, $p<.0001$ for level of experience.

\section{Discussion}

This study found that the postexperience advertising's overshadowing effect that occurred just prior to recall in 
experiment 1 was reinstated after a week's decay of the original taste experience, reversing the negative shift observed in experiment 2 . It appears that the postexperience is working at the reconstructive stage of recall rather than as a source confusion of the information at encoding.

\section{GENERAL DISCUSSION}

The present research provides substantial evidence that consumer recall of past experience is subject to distortion and can be guided by marketing communications. In a series of two experiments and one follow-up study it was shown that advertising received after a direct product experience altered consumers' recollection of both objective sensory and affective components of that experience. In experiment 1, postexperience advertising was found to alter memories within a short time frame - that even unfavorable experiences could be reconstructed to be more favorable in retrospect. In experiment 2 , the findings of postexperience advertising on memory were found to translate into a more typical consumer task of consideration and choice and influenced recollection even a week after the experience. The influences of the postexperience advertising were found to dissipate somewhat over time, however. In the follow-up study, it was found that advertising presented a week after the experience could reinstate the postexperience advertising effects, making evaluations more favorable in recall. It is proposed that the postexperience advertising exerted two levels of effect on memory: immediate overshadowing, where the imagery of the advertising results in less accurate identifications and fewer negative thoughts; and more enduring, as part of developing the brand schema for the new Orange Grove product.

This new reconstructive nature of memory has implications for those interested in how marketers can guide consumer learning (and relearning) from experience, modeling the effects of consumer knowledge, and the role of remembering as an experience.

\section{Learning from Experience}

Learning is to "gain knowledge, understanding or skill by study, instruction or experience'" (cf. Arbib, Conklin, and Hill 1987, p. 99). The insignificant role of direct experience in consumers' learning processes has been attributed to marketing communications that create expectations which shape the way consumers subsequently learn from those experiences (Hoch and Deighton 1989). Advertising that works in this manner can transform consumer experiences to become something beyond the objective evidence at hand.

Learning can also occur incidentally, without such a conscious quest for information. In fact, advertising information may be most likely to influence consumers' experience when they are not aware of its influence. Loftus and Pickrell (1995, p. 720) stated, "New information invades us, like a Trojan horse, precisely because we do not detect its influence." The ease to which the recalled information comes to mind may provide a cue to its validity (Menon, Raghubir, and Schwarz 1995) even if it differs from the actual evidence. In the present experiments, the postexperience influence altered memories, whereby consumers genuinely believed that their past had been as the advertising suggested.

The complex interplay between memory and the external environment determines how consumers perceive their present realities. Because the limbic system is highly creative and fantasy prone (Calvin 1990), recent imagings can distort how experiences are both perceived and remembered. "Perception is not a one-directional process in which stimuli cause 'brain events' that in turn get converted into an internal experience of an outside occurrence. Stimuli cause 'brain events,' but the way in which those events are coded depends partly on what the brain expects and remembers" (Cohen 1996, p. 47).

Cognitive science has moved away from a system where the central computational structure is the manipulation of discrete symbols (e.g., as in information integration) to a dynamic framework called connectionism. Connectionists view cognition not as the manipulation of symbols but rather as a complex mind-brain relationship inspired by the nervous system. The basic idea is that there is a network of connected nodes, each with an activation level, which is able to excite or inhibit other nodes. Because learning is seldom simply substitutability of a new concept, within this connectionist architecture these concepts can grow in meaning and complexity over time as each is tied to other concepts forming richer networks of associations (Arbib et al. 1987).

Connectionist models attempt to take into account the brain's complex interactions between encoding, storage, and retrieval; thus they can be used to model both the effects of prior knowledge or associations on new learning instances and the reconstructive properties of memory found in the present investigation. According to Bruner (1964, p. 4), “Once a child has succeeded in internalizing language as a cognitive instrument, it becomes systematically used to transform the regularities of experience with greater flexibility and power than before." The acquisition of advertising terms demonstrates how brand schemas can evolve over time.

\section{Remembering as an Experience}

Remembering is an emergent experience incapable of meaningful reduction; memories do not exist for the rememberer without retrieval (Endel Tulving, in conversation with Michael Gazzaniga [1997]). This complex configuration occurs in the limbic system, an emotional center of the brain known for providing a sense of conviction that people attach to their ideas and beliefs (LeDoux 1996). The present research attempted to uncover the phenomenological aspects of remembering along with objective evidence demonstrating advertising's intrusion in consumers' memory. The level of consciousness associ- 
ated with memory retrieval has not received any attention in the marketing literature, and consciousness itself has only recently become a view cognitive scientists have considered worthy of investigation (Tulving 1985). However, in the present investigation it was a means of assessing how well consumers internalized the advertising information. Because source attribution comes after the reconstruction process (Johnson, Hashtroudi, and Lindsay 1993 ), people may be biased toward believing the product of their memory retrieval came only from their personal product experience, rather than acknowledging other sources participating in its conception, such as advertising.

\section{Future Research and Limitations}

This is the first investigation of memory reconstruction in the consumer domain. There are many moderating influences upon the reconstruction process and probably still more that will be found to exist within the complex consumer environment. These can be grouped into three broad categories: individual differences, message characteristics, and type of experience. This present research was conducted on students - a relatively homogeneous group with similar backgrounds. This age group is also at the peak of cognitive and memory capability, and we might expect greater effects among older and younger subjects. Since these latter groups are socially protected classes, researchers in social policy may wish to look at memory distortion as an area needing new guidelines for there is a fine line between suggestion and deception. One might imagine that elderly people are particularly susceptible to marketing intended to alter memories, since they often enjoy and take comfort in reminiscence. There may be certain types of messages that are more effective in guiding the reconstruction process, such as transformational writing versus more objective wording (Puto and Wells 1988). Future research should investigate the "timing' of these message presentations in full experimental designs. And, while the product chosen for this experiment was thought to be one that is particularly experience driven, there may be categories for which distortion in memory will be greater or lesser. Future research is needed to address these factors and to investigate how the connectionist architecture might be incorporated into the study of consumer learning.

\section{[Received July 1997. Revised August 1998. Robert E. Burnkrant served as editor, and Richard F. Yalch served as associate editor for this article.]}

\section{REFERENCES}

$\rightarrow$ Alba, Joseph and Lynn Hasher (1983), "Is Memory Schematic?" Psychological Bulletin, 93 (March), 203-231.

Allison, Ralph I. and Kenneth P. Uhl (1964), "Influence of Beer Brand Identification on Taste Perception,' Journal of Marketing Research, 1 (August), 36-39.
Anderson, Norman H. (1991), Contributions to Information Integration Theory, New York: Academic Press.

Arbib, Michael A., E. Jeffrey Conklin, and Jane Hill (1987), From Schema Theory to Language, Oxford: Oxford University Press.

Bartlett, Frederic C. (1932), Remembering, Cambridge: Cambridge University Press.

Bower, Gordon H., John B. Black, and Terrence J. Turner (1979), "Scripts in Text Comprehension and Memory," Cognitive Psychology, 11 (April), 177-220.

Bruner, Jerome (1964), "The Course of Cognitive Growth," American Psychologist, 19 (January), 1-15.

Burke, Raymond R. and Thomas K. Srull (1988), "Competitive Interference and Consumer Memory for Advertising," Journal of Consumer Research, 15 (June), 55-68.

Calvin, William H. (1990), The Cerebral Symphony, New York: Bantam Books.

Cohen, David (1996), The Secret Language of the Mind, San Francisco: Chronicle Books.

Cytowic, Richard L. (1993), The Man Who Tasted Shapes, New York: Putnam.

Donaldson, Wayne (1996), "The Role of Decision Processes in Remembering and Knowing," Memory and Cognition, 24 (July), 523-533.

Fazio, Russell H., Mark P. Zanna, and Joel Cooper (1978), "Direct Experience and Attitude Behavior Consistency: An Information Processing Perspective,' Personality and Social Psychology Bulletin, 4 (Winter), 48-51.

Feldt, Leonard S. (1993), Design and Analysis of Experiments in the Behavioral Sciences, Iowa City: Iowa Testing Programs.

Garry, Maryanne, Charles G. Manning, Elizabeth F. Loftus, and Steven J. Sherman (1996), "Imagination Inflation: Imagining a Childhood Event Inflates Confidence That It Occurred,' Psychonomic Bulletin and Review, 3, 208214.

Gazzaniga, Michael S. (1997), Conversations in the Cognitive Neurosciences, Cambridge, MA: MIT Press.

Greene, Edith, Marlene S. Flynn, and Elizabeth F. Loftus (1982), "Inducing Resistance to Misleading Information,' Journal of Verbal Learning and Verbal Behavior, 21 (April), 207-219.

Hoch, Stephen J. and John Deighton (1989), "'Managing What Consumers Learn from Experience,' Journal of Marketing, 53 (April), 1-20.

and Young-Won Ha (1986), "Consumer Learning: Advertising and the Ambiguity of Product Experience," Journal of Consumer Research, 13 (September), 221233.

Holbrook, Morris B. and Elizabeth C. Hirschman (1982), "The Experiential Aspects of Consumption: Consumer Fantasies, Feelings and Fun,' Journal of Consumer Research, 9 (September), 132-140.

Huber, Joel and John McCann (1982), "The Impact of Inferential Beliefs on Product Evaluations,' Journal of Marketing Research, 19, (August), 324-333.

Johnson, Hollyn M. and Colleen M. Seifert (1994), "Sources of the Continued Influence Effect: When Misinformation in Memory Affects Later Inferences,' Journal of Experimental Psychology: Learning, Memory and Cognition, 20 (November), 1420-1436.

Johnson, Marcia K., Shahin Hashtroudi, and D. Stephen Lindsay (1993), "Source Monitoring," Psychological Bulletin, 114 (July), 3-28. 
LeDoux, Joseph E. (1996), The Emotional Brain, New York: Simon \& Schuster.

Levin, Irwin P. and Gary J. Gaeth (1988), "How Consumers Are Affected by the Framing of Attribute Information before and after Consuming the Product,' Journal of Consumer Research, 15 (December), 374-378.

Lindsay, D. Stephan (1993), "Eyewitness Suggestibility," Current Directions in Psychological Science, 2 (June), 86-89.

Loftus, Elizabeth F. (1977), "Shifting Human Color Memory," Memory and Cognition, 5 (November), 696-699.

- (1979), "Reactions to Blatantly Contradictory Information,' Memory and Cognition, 7 (September), 368 374 .

- (1982), "Memory and Its Distortions," from the G. Stanley Hall lecture series, Washington, DC: American Psychological Association.

- Karen Donders, Hunter G. Hoffman, and Jonathon W. Schooler (1989), "Creating New Memories That Are Quickly Assessed and Confidently Held,' Memory and Cognition, 17 (September), 607-616.

_ and Jacqueline E. Pickrell (1995), “The Formulation of False Memories,' Psychiatric Annals, 25 (December), $720-725$.

Lynch, John G., Dipankar Chakravarti, and Anusree Mitra (1991), "Contrast Effects in Consumer Judgments: Changes in Mental Representations or in the Anchoring of Rating Scales?', Journal of Consumer Research, 18 (December), 284-297.

and Thomas K. Srull (1982), "Memory and Attentional Factors in Consumer Choice: Concepts and Research Methods," Journal of Consumer Research, 9 (June), 1837.

McCloskey, Michael and Maria Zaragoza (1985), “Misleading Postevent Information and Memory for Events: Arguments and Evidence against Memory Impairment Hypotheses,' Journal of Experimental Psychology: General, 114 (March), 1-16.

Melcher, Joseph M. and Jonathon W. Schooler (1996), "The Misrememberance of Wines Past: Verbal and Perceptual Expertise Mediate Verbal Overshadowing Effect,' Journal of Memory and Language, 35 (April), 231-245.

Menon, Geeta, Priya Raghubir, and Norbert Schwarz (1995), "Behavioral Frequency Judgments: An Accessibility-Diagnosticity Framework,' Journal of Consumer Research, 22 (September), 212-228.

Merzenich, Michael M. and Richard C. deCharms (1996), "Neural Representations, Experience, and Change," in The Mind-Brain Continuum, ed. Rodolfo Llinas and Patricia S. Churchland, Cambridge, MA: MIT Press, 61-82.

Metcalfe, Janet (1990), "Composite Holographic Associative Recall Model (CHARM) and Blended Memories in Eyewitness Testimony,' Journal of Experimental Psychology: General, 119 (June), 145-160.

Neisser, Ulric (1967), Cognitive Psychology, New York: Appleton-Century-Crofts.
- (1982), Memory Observed, San Francisco: W. H. Freeman.

Olson, Jerry C. and Phillip A. Dover (1979), "'Disconfirmation of Consumer Expectations through Product Trial,' Journal of Applied Psychology, 64, 179-189.

Payne, David G., Michael P. Toglia, Jeffrey S. Anastasi (1994), "Recognition Performance Level and the Magnitude of the Misinformation Effect in Eyewitness Memory,' Psychonomic Bulletin and Review, 1 (September), 376-382.

Pechmann, Cornelia and S. Ratneshwar (1992), "Consumer Covariation Judgments: Theory or Data Driven?' Journal of Consumer Research, 19 (December), 373-386.

Pezdek, Kathy and Joelle Greene (1993), “'Testing Eyewitness Memory: Developing a Measure That Is More Resistant to Suggestibility,' Law and Human Behavior, 17 (June), $361-369$.

Puto, Christopher P. and William D. Wells (1988), “'Informational and Transformational Advertising: The Differential Effects of Time,', in Advances in Consumer Research, Vol. 11, ed. Thomas C. Kinnear, Provo, UT: Association for Consumer Research, 638-643.

Schacter, Daniel L. (1996a), "Illusory Memories: A Cognitive Neuroscience Perspective," colloquium paper, Procedures National Academy of Sciences, 93, 13527-13533.

(1996b), Searching for Memory, New York: Basic Books.

Schwarz, Norbert (1996), Cognition and Communication, Mahwah, NJ: Erlbaum.

Smith, Robert E. (1993), “Integrating Information from Advertising and Trial: Processes and Effects on Consumer Response to Product Information,' Journal of Marketing Research, 30 (May), 204-219.

and William R. Swinyard (1983), “Attitude-Behavior Consistency: The Impact of Product Trial versus Advertising," Journal of Marketing Research, 20 (August), 257267.

Snodgrass, Gay (1997), "The Memory Trainers,"' in Mind and Brain Sciences in the Twenty-First Century, ed. Robert L. Solso, Cambridge, MA: MIT Press.

Stayman, Douglas M., Dana L. Alden, and Karen H. Smith (1992), "Some Effects of Schematic Processing on Consumer Expectation and Disconfirmation Judgments," Journal of Consumer Research, 19 (September), 240255.

Thomson, D. M. and Endel Tulving (1970), “Associative Encoding and Retrieval: Weak and Strong Cues,' Journal of Experiment Psychology, 86 (November), 255-262.

Tulving, Endel (1985), 'Memory and Consciousness,' Canadian Psychologist, 26 (January), 1-12.

West, Patricia M., Christina L. Brown, and Stephen J. Hoch (1996), "Consumption Vocabulary and Preference," Journal of Consumer Research, 23 (September), 120135.

Wright, Alice A. and John G. Lynch (1995), “Communication Effects of Advertising versus Direct Experience When Both Search and Experience Attributes Are Present,', Journal of Consumer Research, 21 (March), 708-718. 